\title{
Time-place learning in the eight-arm radial maze
}

\author{
MATTHEW J. PIZZO and JONATHON D. CRYSTAL \\ University of Georgia, Athens, Georgia
}

\begin{abstract}
Rats $(n=4)$ searched for food on an eight-arm radial maze. Daily 56-min sessions were divided into eight 7-min time zones, during each of which a different location provided food; locations were randomized across subjects before training. The rats obtained multiple pellets within each time zone by leaving and returning to the correct location. Evidence that the rats had knowledge about the temporal and spatial features of the task includes the following. The rats anticipated locations before they became active and anticipated the end of the currently active locations. The rats discriminated currently active locations from earlier and forthcoming active locations in the absence of food transition cues. After the rats had left the previously active location, they visited the next correct location more often than would be expected by chance in the absence of food transition cues. The rats used handling or opening doors as a cue to visit the first location and timed successive 7-min intervals to get to subsequent locations.
\end{abstract}

A growing body of literature has characterized two timing mechanisms by which animals adapt their behavior to the temporal features of the environment. According to Gibbon, Fairhurst, and Goldberg (1997), interval-timing systems are used to anticipate events that occur at an arbitrary time after an event. This type of timing mechanism is used for timing relatively short intervals. A characteristic of this timing system is that it has a low level of precision (i.e., it is characterized by high variability). In contrast, circadian timers are used to anticipate events that occur with daily regularity (i.e., approximately $24 \mathrm{~h}$ ). These timers are entrained to a limited set of events that have approximately a 24-h interval between successive occurrences and have higher precision relative to an interval timer (Gibbon et al., 1997).

Research on time-place learning is concerned with animals' use of spatial and temporal information to exploit biologically significant events (e.g., food, mates, and prey). Time-place learning has been documented in a variety of species (Daan \& Koene, 1981; Pizzo \& Crystal, 2002; Saksida \& Wilkie, 1994; Wahl, 1932). For example, Biebach, Gordijn, and Krebs (1989) evaluated the ability of garden warblers to learn about predictable changes in the location of food as a function of time. The birds were housed in an apparatus that consisted of five rooms; four rooms were connected to a central area. Each of the four rooms pro-

The data were presented at the 10th Annual Meeting of the International Conference on Comparative Cognition, Melbourne, FL, March 2003. This work was supported by National Institute of Mental Health Grants MH64799 and MH61618 to J.D.C. This research was completed in partial fulfillment of a doctoral degree to M.J.P. at the University of Georgia. The raw data (time of occurrence of each stimulus and response) are available at www.uga.edu/animal-cognition-lab. We thank Kenneth W. Maxwell for technical assistance during the course of this study. Correspondence concerning this article should be addressed to M. J. Pizzo or J. D. Crystal, Department of Psychology, University of Georgia, Athens, GA 30602-3013 (e-mail: mpizzo@uga.edu; jcrystal@ uga.edu). vided food for a different 3 -h interval during a 12-h daily session. The birds were able to restrict most of their visits to the correct room during each interval of the session.

The use of a circadian timing mechanism has been shown in several time-place studies. The successive intervals for food availability in time-place tasks based on circadian mechanisms are typically measured in the range of hours. One example of this is a study by Mistlberger, de Groot, Bossert, and Marchant (1996), in which they tested rats in a T-maze. The rats could receive food when they leverpressed at one end of the maze in the morning and at the other end in the afternoon. The morning and the afternoon shifts were separated by $7 \mathrm{~h}$. Several manipulations verified the use of a circadian mechanism. Omitting sessions or inverting the light-dark cycle did not disrupt their performance. This rules out the hypotheses that the rats were using an alternation strategy, timing with respect to light cues, or timing on the basis of a physiological state (e.g., hunger).

Additional evidence to support time-place learning based on a circadian mechanism comes from a study by Biebach, Falk, and Krebs (1991). They sought to identify the mechanism that allowed garden warblers to complete the time-place task in the study by Biebach et al. (1989) described above. When the birds were tested in constant light conditions, they continued to visit the rooms in the correct sequence for 1-6 days. When the light cycle was phase advanced, activity at each location moved forward but was significantly different from that predicted by timing the interval from light onset to feeding. On test days in which the light cycle was delayed, there was no significant change in their activity at each location. These results support the hypothesis that the birds were utilizing a circadian timing mechanism to complete the task. The possibility that the birds were timing with respect to the 4-h intervals in the experiment was ruled out by blocking access to all rooms for $4 \mathrm{~h}$. When access to the rooms resumed, they visited the room associated with the current time of day (Krebs \& Biebach, 1989). 
Time-place tasks have also been completed on the basis of interval-timing mechanisms. The duration of food availability in these tasks is much shorter than that in a circadian time-place task. Carr and Wilkie (1998) trained rats in a time-place task in which they were required to press one of four levers in an operant chamber to receive a reward. Each lever provided reinforcement at one of four different times. There were three groups of rats, each tested with a different interval. For the three groups of animals, reinforcement was available at each of the four locations for 4,6 , or $8 \mathrm{~min}$, respectively. For example, for one group, leverpressing at the four locations was available for 4,4 , 4 , and 4 min, respectively, during a 16 -min session. The rats restricted most of their responses to the correct lever at the appropriate time. The variability in the distributions of response rates as a function of time, was constant at each lever. Similarly, the precision with which the rats switched from one lever to the next was constant as the session progressed. This was interpreted as evidence for the timing of successive intervals, rather than the timing of one interval equal to the length of the session. In addition, responding during each task (i.e., 4, 6, or 8 min) obeyed Weber's law. Weber's law is a hallmark of interval timing (e.g., Gibbon, 1991). It states that the spread (i.e., width) of a distribution is proportional to the interval being timed. In other words, the amount of variability in responding throughout the interval should be greatest in the 8-min task and proportionately less in the 6- and 4-min tasks. The observation that the data could be superimposed when plotted in relative time (i.e., elapsed time divided by 4,6 , or $8 \mathrm{~min}$ for the three groups, respectively) is consistent with the use of an interval-timing mechanism; however, for an example of data that do not superimpose in a short-interval timeplace task, see Crystal and Miller (2002).

The studies reviewed above indicate that there is evidence for interval and circadian time-place learning. However, there are several limitations evident in the time-place literature. The conditions under which different types of time-place learning occur have not been established. It is not known what lengths of time are applicable to interval and circadian time-place learning. Although it is unlikely that a circadian oscillator can be used to time short intervals, there is evidence that (1) periods below the conventional entrainment boundary can be anticipated (Crystal, 2001, 2003, 2004), (2) circadian phase can be used to time intervals as short as $1 \mathrm{~h}$ (Aschoff, 1984, 1985, 1989, 1993, 1998; Aschoff \& Daan, 1997), and (3) phases as short as 7-11 min can be discriminated (Frisch, 1967). Circadian time-place learning has not been documented with intervals shorter than $3 \mathrm{~h}$ (Biebach et al., 1989). On the other hand, time-place learning based on an interval-timing mechanism has not been shown with intervals longer than $8 \mathrm{~min}$ (Carr \& Wilkie, 1998). Another limitation is that timeplace studies have been restricted to tasks involving relatively few (e.g., two to four) locations (Biebach et al., 1989; Carr \& Wilkie, 1997; Mistlberger et al., 1996; Pizzo \& Crystal, 2002).

A desirable feature of a time-place study is that it be designed so that either circadian or interval-timing mecha- nisms could be used to solve the task. If both time of day and interval-timing mechanisms are available, tests can be designed to identify which mechanism the animal uses to restrict its visits to the correct locations and times. This feature has generally not been included in previous timeplace experiments. For example, Carr and Wilkie (1998) randomly tested rats daily in a random order within a 3-h window of time. Therefore, time of day was rendered irrelevant. In order for both timing mechanisms to be made available, it is necessary to test the animals at the same time each day.

The present work was conducted in order to address some of the limitations in the time-place literature by making available both interval and circadian mechanisms. Eight locations were used to determine whether timeplace learning is limited to four locations. Rats were trained in a fixed order in an eight-arm radial maze. Each location provided reinforcement for a different 7-min interval during a 56-min session. Testing was conducted at precisely the same time each day so that food availability could be predicted by a circadian mechanism. We assessed knowledge of time and place (Experiments 1 and 2). We identified four potential mechanisms that could be used to solve the time-place task, and we systematically tested each mechanism (Experiments 3A-3D). The rats used door opening or handling as a cue(s) to go to the first location and visited subsequent locations by timing successive 7 min intervals.

\section{EXPERIMENT 1}

\section{Method}

Subjects. Four male Long-Evans rats were obtained from Harlan (Madison, WI). The rats were given unlimited access to 5001 Rodent Diet (Lab Diet, Brentwood, MO) for 1 week, followed by $20 \mathrm{~g}$ for 1 day, and $15 \mathrm{~g}$ per day thereafter. They were given unlimited access to water, except during the experimental sessions. The rats were individually housed in clear plastic cages lined with bedding (Andersons, Bed'o cobs, Maumee, $\mathrm{OH}$ ). The colony's light onset and offset occurred at 0710 and $1905 \mathrm{~h}$, respectively. The rats were 140 days old and weighed $313 \mathrm{~g}$ at the beginning of the experiment; they weighed $350 \mathrm{~g}$ at the end of the experiment.

The rats participated in a pilot version of the present experiment for 44 sessions, in which the animals were exposed to the time-place task described below, with variations in the use of the guillotine doors to limit access to the arms. The pilot demonstrated that it was not possible to automatically and reliably close the doors behind the rat as it entered the hub.

Apparatus. Testing was conducted in an eight-arm radial maze. The central hub (MED Associates, ENV-538) consisted of a white polypropylene octagonal base ( $28.6 \mathrm{~cm}$ in diameter, $11.4-\mathrm{cm}$ sides $)$, metal walls ( $33.3 \mathrm{~cm}$ high), and a clear polycarbonate lid. The arms radiated from the center hub, with equal spacing between each arm. Each arm was $76.2 \mathrm{~cm}$ long and $8.9 \mathrm{~cm}$ wide with clear polycarbonate walls $(17.5 \mathrm{~cm}$ high) and was covered with strips of polycarbonate of the same length. The central hub was equipped with eight guillotine doors (MED Associates, ENV-540). A food trough (MED Associates, ENV-200R1M) was located at the end of each arm. A photobeam (MED Associates, ENV-254) was situated $1 \mathrm{~cm}$ inside each of the food troughs ( $1 \mathrm{~cm}$ from the trough bottom) to detect head entries. A pellet dispenser (MED Associates, ENV-203) was positioned behind each food trough. Additional photobeams were located in each arm at 3.8 and $5.1 \mathrm{~cm}$ from the guillotine doors. 
The maze was positioned inside an octagonal frame. The octagonal frame (298 cm in diameter), consisting of PVC tubing $(130 \mathrm{~cm}$ long on each side), was suspended from the ceiling with wires. This frame included curtains that were retracted throughout the experiment so that extra-maze cues were visible. The maze was positioned on stools $81.3 \mathrm{~cm}$ above the floor. There were several other objects in the room (i.e., a desk, a chair, a sink, shelves, and posters) that remained in fixed locations throughout the experiment. White noise $(67 \mathrm{~dB})$ was used to mask outside noises. A 500-MHz computer in an adjacent room, running MED-PC for Windows (Version 1.15), controlled experimental events (i.e., guillotine doors and food) and recorded photobeam interruptions with $10-\mathrm{msec}$ resolution. A video monitor was positioned in the ceiling above the center of the maze to permit observation of the rats from an adjacent room.

Procedure. Pretraining consisted of 4 days in which four 45-mg pellets (Noyes Precision pellets, Improved Formula A/I, Research Diets, New Brunswick, NJ) were placed along each arm, and one pellet was placed in each food trough. The rats were individually placed in the maze for approximately $20 \mathrm{~min}$ per day. When it had eaten all the pellets, the subject was removed, and the next rat was placed in the maze.

In the time-place task, each session was $56 \mathrm{~min}$ in duration. The session was divided into 8 time zones, each with a duration of $7 \mathrm{~min}$; no stimuli signaled the transition across time zones. During a given time zone, one of the arms of the maze was active and provided food. The order of active locations was randomized for each rat prior to the start of the experiment. The order was used consistently for that rat throughout the experiment. For example, for 1 of the rats, food was available sequentially at the following locations: 5, 2, 4, 7, 1, 8, 6, and 3 . When a location was active, food was available intermittently, using the following schedule. Food was delivered contingent on the breaking of the photobeam inside the active food trough. After a pellet was delivered, no additional food was available until the rat approached the hub. Approaching the hub was defined as interruption of the photobeam adjacent to the hub on the current arm. An approach to the hub did not require that the rat exit the current arm, although the rats frequently exited an arm after approaching the hub. The approach initiated a $10-\mathrm{sec}$ intertrial interval (ITI). At the end of each ITI, food was again available at the currently active location, following the schedule described above. The rats were able to obtain multiple pellets during each time zone. They were free to visit any of the locations (active or inactive) at any time but could not receive reinforcement at the active location until the ITI had ended and the appropriate food trough photobeam had been interrupted.

The time of daily testing was carefully controlled to ensure that interval and circadian cues were consistently associated with food availability. Sessions started at a fixed time $\pm 30 \mathrm{sec}$ for each subject, using a clock (Atomic Moon Phase Clock, Model 86715) that received a radio transmission from a Cesium-133 clock operated by the National Institute of Standards and Technology. If it was not possible to start the rat's session according to the schedule described above, the rat was not tested on that day; a rat's session was canceled on four occasions. The standard deviation of start times was $5 \mathrm{sec}$. Daily testing of the first rat began at $1400 \mathrm{~h}$. After each shift, the rat was returned to the colony, and the next rat was tested in a consistent order each day. The rats received a daily postsession ration at approximately $1800 \mathrm{~h}$. The amount of food in the postsession ration was adjusted on the basis of the amount of food in the session to maintain daily consumption at $15-20 \mathrm{~g}$. Testing was conducted 5-7 days per week. The arms of the maze were washed daily with a diluted solution of Nolvasan (Fort Dodge Animal Health, Fort Dodge, IA). Acquisition consisted of 61 sessions.

Data analysis. The dependent variable was the frequency of visits to each of the eight locations. A visit was defined as the first interruption of a food trough photobeam following the interruption of the photobeam closest to the central hub of that arm. This definition of a visit excluded any additional interruptions of the photobeam at the food trough while the pellet was being consumed. When a photobeam near the hub was interrupted, it was followed by a photobeam interruption at a food trough $87.4 \%$ of the time, on average. All photobeam interruptions were recorded.

Throughout this article, the criterion for statistical significance is .05 .

\section{Results and Discussion}

Acquisition. Figure 1 plots the proportion of visits that occurred at correct locations (visits at the correct location divided by the sum of visits to all locations averaged across the eight locations) as a function of five-session blocks of acquisition. The mean proportion correct in the first ses-

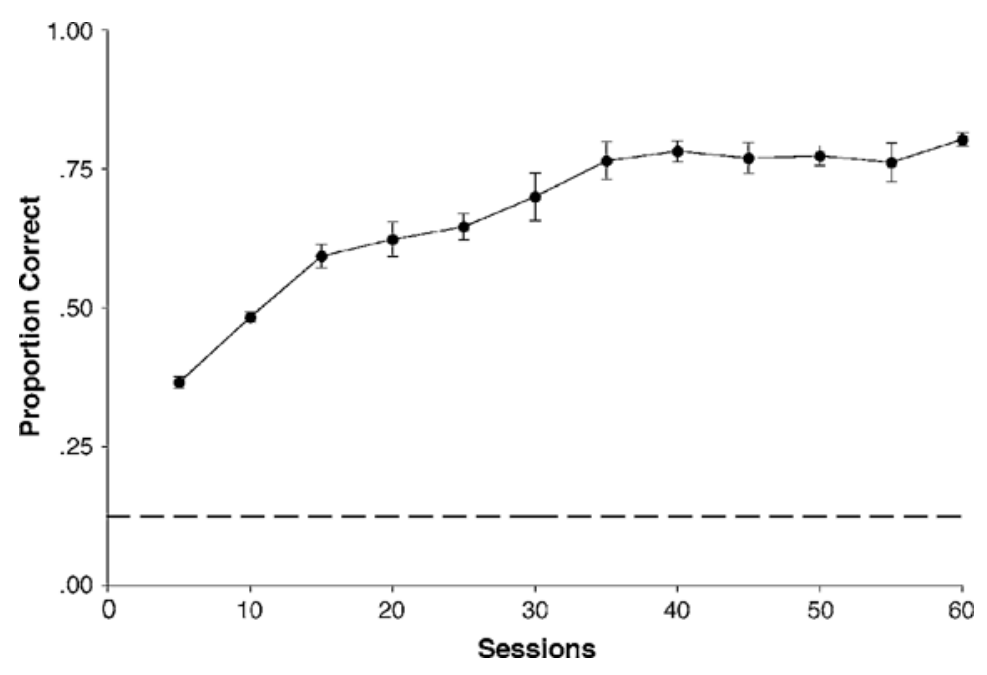

Figure 1. Mean proportion of visits at the correct location plotted as a function of blocks of five sessions in Experiment 1. The data are means across the eight active locations from the eight time zones. The dashed line indicates the proportion correct expected by chance. Error bars represent $\pm 1 S E$. 
sion was .20 $(S E=.04)$; although the initial level of performance might represent an overestimate, due to prior experience with the task in the pilot study, performance on the first session was not significantly different from the chance level of .125. The high terminal level of performance documents that the rats were directing most of their visits to the temporally correct location. An analysis of variance (ANOVA) with session as a repeated measure revealed that there was a significant effect of sessions $[F(11,33)=$ $13.39, p<.01]$. The proportion correct reached a plateau during the last 30 sessions; therefore, all subsequent analyses were conducted on the last 30 sessions.

Time-place performance. Figure 2 plots the response rate as a function of time since the start of the session at each location. The rats allocated the majority of their responses to the active location during each time zone. Responses at each location reached a plateau early in the time zone shortly after each location became active. An exception to this is observed at Location 1; responses did not reach plateau levels until approximately the middle of Time Zone 1. Immediately after a location became inactive, response rates declined within approximately $1 \mathrm{~min}$.

Small peaks in response rate occurred at the start of new time zones. The peaks occurred at 7-min intervals, at each location that had not yet become active in the session (i.e., note that peaks did not occur after the primary visits to each location on the right side of Figure 2). These observations suggest that (1) the rats searched multiple locations when an active location ceased to provide food and (2) the rats remembered which locations had already been
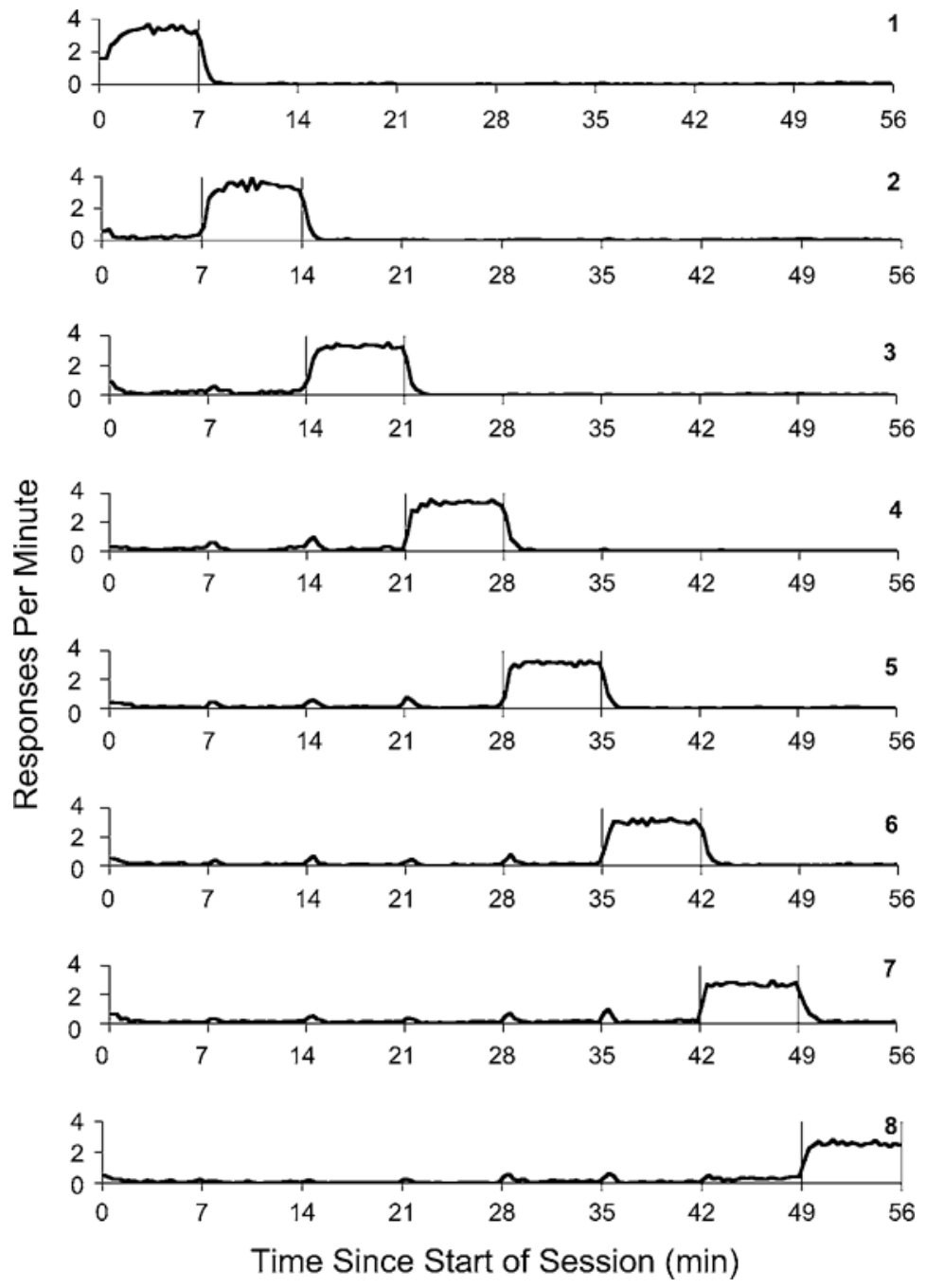

Figure 2. Responses per minute plotted as a function of time since the start of the session at each location during the last 30 sessions in Experiment 1. Each panel displays data from one location; the location is indicated by the number in each panel. Food was available between the times indicated by vertical lines in each panel. 
active in the current session (i.e., working memory), thereby restricting the search to locations that were scheduled to provide food.

The high response rates at the active locations during each time zone in Figure 2 indicate that the rats effectively restricted the majority of their visits to locations that were currently providing food. However, an animal could have produced this pattern of data by inspecting locations until the currently active location had been visited, at which point the animal could have continued to visit the active location until food was no longer available at that location. We distinguish between a win-stay, lose-shift hypothesis with no knowledge of time and place and a win-stay, lose- shift hypothesis with knowledge of time and place. According to the former hypothesis, the rats are expected to search locations randomly after leaving a previously active location. According to the latter hypothesis, the rats are expected to adjust their search behavior before experiencing changes in food availability. In the next several sections, we will test for knowledge of time and/or place.

Temporal anticipation of spatial location. An animal that used a win-stay, lose-shift strategy without any knowledge of time or place would not be expected to anticipate the arrival of food before it was available. In contrast, an animal might time each time zone by utilizing information about the sequence of active locations, in addition to depending on the food cues of a win-stay, lose-shift strategy. One method by which to test the hypothesis that the animal has no knowledge of time and place is to examine anticipation of the soon-to-be active location. An animal that relies on a win-stay, lose-shift strategy without the use of temporal or spatial information about the time-place contingencies would not anticipate the next active location. In contrast, an animal that was timing the current time zone would be able to visit the next location before any food is available at that location. Anticipation of the location that will be active in the next time zone can be documented by a higher terminal response rate at the end of a time zone, relative to the rate earlier in the time zone at that location.

Figure 3 plots the response rate at the next active and other inactive locations as a function of time prior to the start of the active location. The data for the next active location were the visits averaged across the prior intervals for Locations 2-8 (i.e., locations that have a time zone to examine prior to the active location). The other inactivelocation data consist of the average of all visits excluding those at the next active and currently active locations. More responses were allocated to the next active location than to the other inactive locations. A peak in response rate is apparent at the beginning of the interval for both types of location; these peaks in response rate correspond to the small peaks in Figure 2 that occurred at the start of new time zones. After the initial peak, the response rates at both locations decreased to a low point during the interval. This was followed by an increase in the function toward the end of the interval at the next active location. The function is relatively flat at the inactive locations for the remainder of the interval. The increase in response rate at the next active location was small (Figure 3), relative to the rate of visiting currently active locations (Figure 2). Therefore, it is difficult to observe relatively small changes in rates in Figure 2.

The data from the last $5 \mathrm{~min}$ of the active interval were subjected to a two-way ANOVA with time as a repeated measure and location as a between measure; the first $2 \mathrm{~min}$ were excluded to remove the initial peak. Response rate was higher at the next active location than at the other inactive locations $[F(1,6)=45.5, p<.01]$. Response rate increased as a function of time $[F(14,84)=6.74, p<.01]$. There was a significant time $\times$ location interaction $[F(14,84)=2.63$, $p<.05]$. This interaction reflects the fact that the propor-

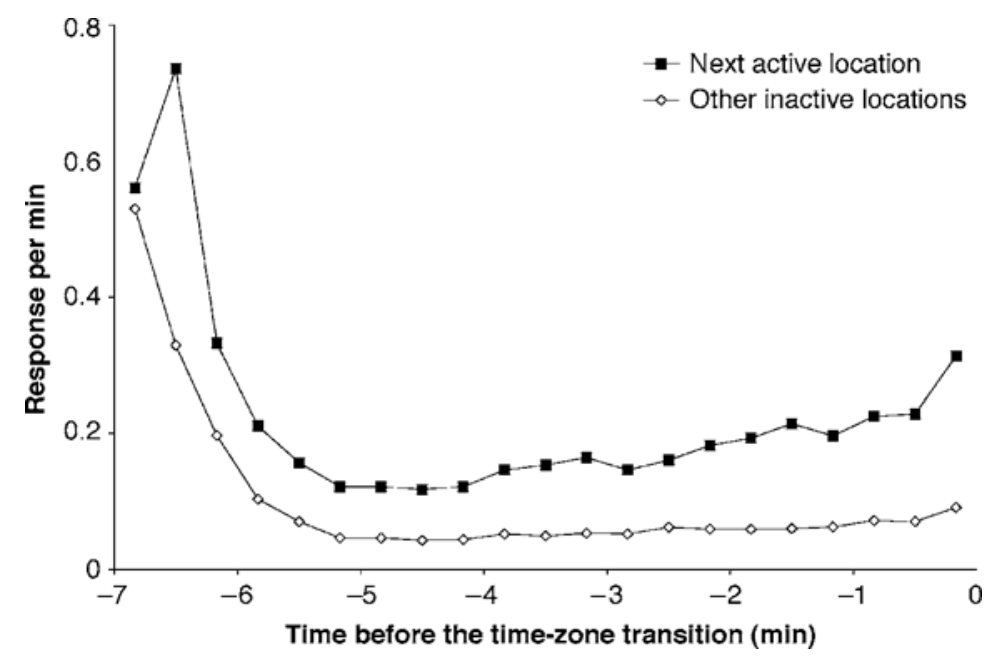

Figure 3. Responses per minute at the next active and inactive locations plotted as a function of time prior to the active interval in Experiment 1. The $x$-axis is plotted in reverse order; zero indicates the start of the next time zone. 
tion of responses allocated to the next active location increased more rapidly as a function of time than did the response rate at the other inactive locations (see Figure 3).

To characterize the nature of the interaction, the data at the next active location were subjected to an ANOVA with time as a repeated measure. This revealed a significant effect of time $[F(14,42)=4.72, p<.01$; estimated effect size, $\omega^{2}=0.73$ ], indicating that the rats anticipated the arrival of food at the next active location before food became available at that location. To evaluate anticipation at the next active interval, we compared the proportion of responses at the next active location at $5 \mathrm{~min}$ prior to the time zone transition versus the proportion during the time just prior to the transition. This contrast revealed a significant effect $[F(1,3)=13.4, p<.05]$.

The data at the other inactive locations were also subjected to an ANOVA with time as a repeated measure. This also revealed a significant effect of time $[F(14,42)=$ $4.17, p<.01]$. Anticipation at the next active interval was assessed by comparing the proportion of responses at the inactive locations at $5 \mathrm{~min}$ prior to the time zone transition versus the proportion during the time just prior to the transition. This contrast was not significant $[F(1,3)=6.84$, $p>.05]$.

The high proportion of responses at both the next active and other inactive locations in Figure 3 corresponds to the search that occurs at the start of time zones at locations that have not yet provided food in the session (next active and other inactive locations have not yet provided a reward during the session). This was described in the section above (see Figure 2). The observation that the response rate increased at the next active location before the end of the time zone suggests that the rats anticipated the end of the 7-min interval at the next location scheduled to provide food. Although the increase in response rate was relatively small, these anticipatory visits occurred while the rats visited the active location, which provided food on an intermittent schedule. Nevertheless, the reliable increase in response rate at the end of the time zone is consistent with the animals' timing the active time zone. The lower, relatively flat function corresponding to the proportion of responses allocated to the other inactive locations suggests that the rats have knowledge of what places will not provide reward during the next interval. In conclusion, the rats anticipated the end of the 7-min interval at the next active location while searching other locations at a lower rate.

Superposition. The observation that the rats were anticipating the arrival of food at the next active location suggests that the rats were timing an interval. However, these data do not indicate what interval they were timing. According to one hypothesis, the animals were timing successive 7-min intervals throughout the session. According to this hypothesis, the rat searched at the next location as the end of the 7-min interval approached. According to a second hypothesis, the rats were timing a cumulative interval with respect to the start of the session (i.e., $7 \mathrm{~min}$ to get to Location 2, $14 \mathrm{~min}$ to get to Location 3 . . 49 min to get to Location 8 ). To test these hypotheses, we separately examined the data prior to active Locations $2-8$ as a function of time (i.e., the individual anticipation functions that were averaged and presented in Figure 3 as the next active location). For each location, we determined the time (in minutes) at which the response rate exceeded $85 \%$ of its maximum rate to provide a quantitative measure of superposition. These data were expressed in relative time by dividing the transition time by a value that was determined by the hypotheses described above. If the rats were timing $7 \mathrm{~min}$ for each location, dividing each transition time by $7 \mathrm{~min}$ should produce values that superimpose (i.e., relative time values that do not vary systematically across locations). Alternatively, if the rats were timing $7,14,21, \ldots 49 \mathrm{~min}$ for Locations $2,3,4, \ldots 8$, respectively, then dividing each transition time by $7,14,21, \ldots 49$ min should produce values that superimpose.

Response rate at Locations 2-8 were examined in the time zone before each location became active. Response rate was expressed as a percentage of the maximum rate at each location. The time (in minutes) at which the percentage of maximum rate crossed $85 \%$ was determined for each subject at each location. The first 2 min of the interval were excluded from this analysis, because this was the point at which a peak occurred at the start of time zones at the forthcoming active locations (see Figures 2 and 3). There were three instances in which multiple points of transition across $85 \%$ of the maximum rate occurred. In these three cases, the data were subjected to a median smooth in order to estimate a single point of transition.

The top panel of Figure 4 plots the relative transition times, using the hypothesis that the rats were timing successive 7-min intervals at Locations $2-8$. There are no apparent trends in the relative transition times with respect to the hypothesis that the rats were timing successive 7-min intervals. The bottom panel of Figure 4 plots the relative transition times, using the hypothesis that the rats were timing with respect to the start of the session (i.e., 7, 14, . . . $49 \mathrm{~min}$ ) for Locations 2-8. Relative transition times decreased as the session progressed. If the rats were timing successive 7-min intervals, we would predict that the points of transition should be constant when divided by 7 but not constant in transition times divided by 7-49. Alternatively, if the rats were timing one cumulative interval throughout the session, the transition times would be constant when divided by 7-49, but not when divided by 7 . ANOVAs were performed on the relative transition times, with location as a repeated measure. There were no significant differences in the transition times divided by 7 at each of the locations in the analysis $[F(6,18)=.75, p>$ $.05]$. This result suggests that transition times divided by 7 superimpose in relative time (Figure 4, top panel). There was a significant difference in the transition times divided by $7-49[F(6,18)=5.67, p<.01]$. This result suggests that these transition times do not superimpose in relative time with respect to timing the entire session (Figure 4, bottom panel). In conclusion, the results of the superposition analysis suggest that the rats were timing successive 7-min intervals at each active location. 

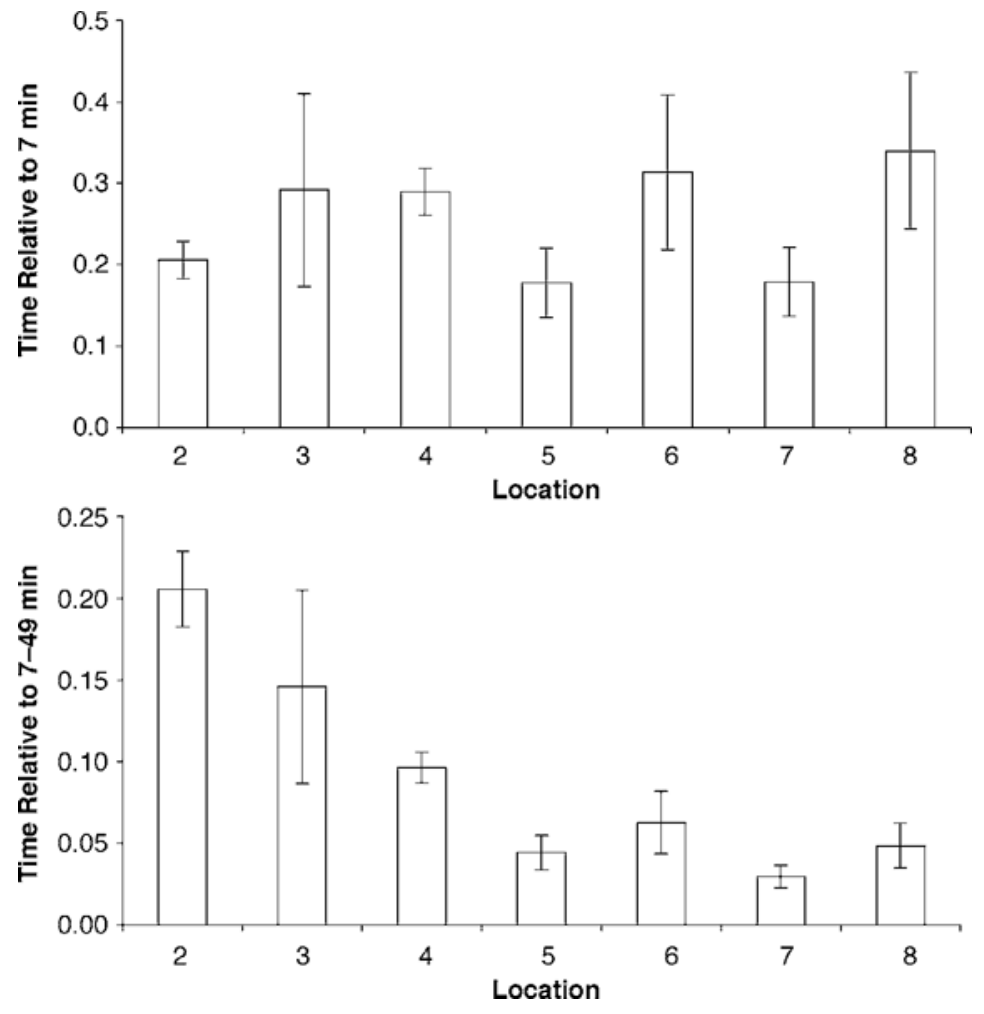

Figure 4. Top: the relative time of transition beyond $85 \%$ divided by 7 at each location is plotted as a function of locations to evaluate the successive 7-min interval hypothesis. Bottom: the relative time of transition beyond $85 \%$ divided by $7-49$ is plotted as a function of locations to evaluate the hypothesis that the rats were timing the session. Error bars represent $\pm 1 S E$.

Counting pellets. It is possible that the rats were counting the number of pellets earned during a given time zone and then moved to the next active location, rather than timing successive 7 -min intervals. If the rats were sensitive to the number of pellets, we would expect to observe a change in the proportion correct as a function of the number of pellets earned during the time zone. To test this hypothesis, we analyzed the proportion correct after few and many pellets while holding time within the time zone constant. We first counted the number of pellets earned in the first $3.5 \mathrm{~min}$ of each trial. Next, we identified trials in which the rats received less or more than the median amount of food. Finally, we identified the proportion of times that they visited the correct location on these lowfood and high-food trials, using the visits that preceded and followed $3.5 \mathrm{~min}$ into the time zone; these proportions were .80 and .88 for low- and high-food trials, respectively. The number of pellets earned and the amount of time at the active location are confounded. In particular, the amount of food earned correlates with the amount of time that was available to earn food. Therefore, the time at which the rats first visited the correct location was used as a covariate in an analysis of covariance on the proportion of correct visits, with low- versus high-food trial types serving as a repeated measure. This analysis revealed that there was no significant difference between these two proportions $[F(1,2)=9.35, p>.05]$. This suggests that the number of pellets earned did not influence the rate of visiting the correct location while holding time constant for the comparison across food quantities. The observation that proportion correct did not change as a function of food should be interpreted with caution. This experiment was not designed to unconfound food and time; these variables could be unconfounded experimentally by manipulating the rate of food presentation.

Anticipation of departure from the currently active location. The fact that the rats anticipated the next active location suggests that they may have been timing the duration of the time zone. Additional evidence for timing may come from observing anticipation of the end of the current time zone (i.e., a decline in visits as a function of time at the currently active location); an animal with no knowledge of time or place would not be expected to anticipate the end of the currently active time zone. Figure 5 displays the proportion of correct visits as a function of time. Initially, the proportion correct increased. After $2 \mathrm{~min}$, the proportion reached its highest point during the time zone. Then the proportion decreased slightly over the next 


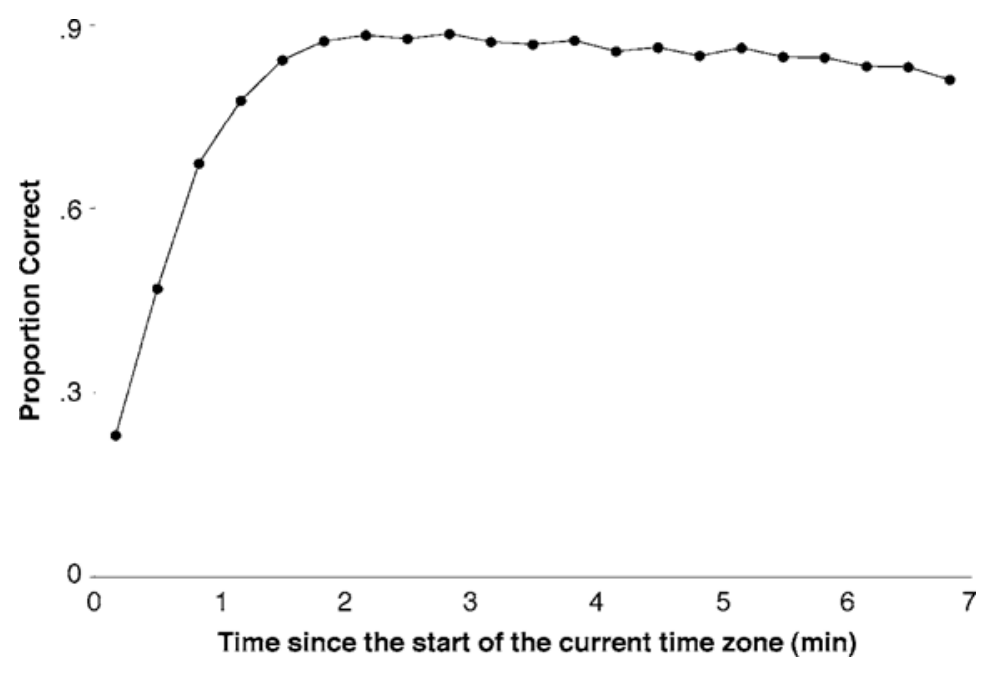

Figure 5. Proportion of correct visits plotted as a function of time since the start of the current time zone in Experiment 1.

$5 \mathrm{~min}$. The decrease in visits at the currently active location was small (Figure 5) relative to the rate of visiting currently active locations. Therefore, it is difficult to observe the relatively small changes in Figure 2.

The data from the last $5 \mathrm{~min}$ of the active interval were subjected to an ANOVA with time as a repeated measure. The first $2 \mathrm{~min}$ of data were excluded to remove the initial increase. The proportion correct decreased as a function of time $[F(14,42)=4.21, p<.05$; estimated effect size, $\left.\omega^{2}=0.44\right]$. The proportion correct declined by .075 . This suggests that the rats anticipated the end of the current time zone. They did this despite food continuing to be available at the active location.

As the end of the time zone approached, the rats searched the currently active location less frequently (Figure 5), while they searched the next active location more frequently (Figure 3). The rats searched other locations while they continued to get food from the active location. Although both changes in response rate were relatively small, they are both consistent with the rats' timing successive 7-min intervals. It is noteworthy that both types of anticipation reflect errors; no food is obtained by visiting the next active location early, and less food is obtained by reducing visits to the currently active location. Therefore, small anticipatory changes in Figures 3 and 5 may reflect a strategy in which the rats check the next active location while minimizing lost opportunities to obtain food at the currently active location. Departures in responding at the active location while food remained present have been documented in previous time-place studies involving interval timing (Carr \& Wilkie, 1998) and circadian timing (Biebach et al., 1991; Biebach et al., 1989; Krebs \& Biebach, 1989).

Spatial choice in absence of food transition cues. The observation of temporal anticipation restricted to the spatially correct location supports the hypothesis that the rats have information about time and place. If the rats were relying exclusively on food transition cues without any knowledge of time and place, then when a rat leaves the previously active location at the start of the new time zone, the visits should occur randomly among the other locations. In contrast, if the rats had knowledge about the next active location, then when a rat leaves the previously active location at the start of the new time zone, the rats would be expected to visit the currently active location more frequently than other locations.

To assess the knowledge about the currently active location, we analyzed the segment of data that occurred immediately after a time zone transition (i.e., before receiving food at the start of a new time zone). The data consisted of the responses that occurred from the start of a new time zone until (1) a visit occurred at the currently active location or (2) a visit occurred at the previously active location. This means that responses were analyzed until the rat either earned food from the currently active location (thereby receiving a food cue) or did not receive food from the location that was previously active (thereby receiving a nonfood cue). The duration of this segment of data was 10.5 sec, on average, and terminated with a nonfood cue on $79 \%$ of the trials.

If a location was currently scheduled to provide reinforcement, it was categorized as currently active. The location that had just ceased to provide reinforcement is referred to as the previously active location. If a location had not yet provided reinforcement during the session, it is referred to as a forthcoming active location. If a location has provided reinforcement before the previously active location, it is referred to as an earlier active location.

Figure 6 plots the proportion of responses at all locations from the start of Time Zones 2-8; a break appears in the $y$-axis so that differences between currently active and forthcoming and earlier active locations can be seen in the same figure in which a high rate occurred for the previously active location. The proportions were calculated separately for each time zone (note that the sum of 


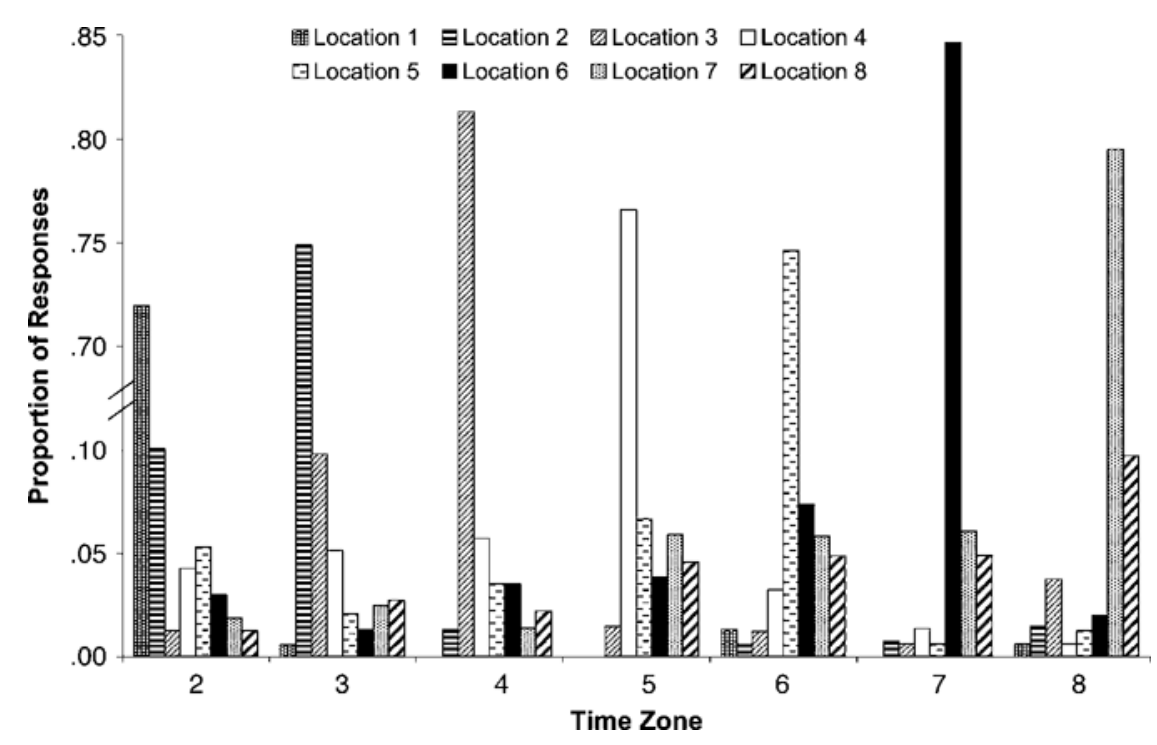

Figure 6. Proportion of responses allocated to each location plotted for each time zone at the time of transition into a new time zone (i.e., before food transition cues were received) in Experiment 1. Note the break in the $y$-axis.

proportions from the eight locations equaled 1.0 at each time zone for each rat). This figure illustrates that a large proportion of responses occurred at the previously active location. The second highest number of responses occurred at the currently active location. The data were subjected to an ANOVA with time zone and location as repeated measures. There was a significant effect of location $[F(7,21)=8.52, p<.01]$, but not of time zone $[F(6,18)=$ $0.63, p>.05]$. The interaction was also significant $[F(42,126)=55.03, p<.01]$. This interaction reflects the fact that the rats visited the previously active location after each time zone transition; this location was different during each time zone transition.
Figure 7 plots the average proportion of responses to each type of location during the session, combining across time zones; a break appears in the $y$-axis so that differences between currently active and forthcoming and earlier active locations can be seen in the same figure in which a high rate occurred for the previously active location.

These data are consistent with the hypothesis that the rats were using nonfood transition cues. On the majority of the trials, the rats stayed at the previously active locations after the time zone transition, thereby obtaining a nonfood cue.

On the subset of trials in which the rats did not experience a nonfood cue, there is evidence that the rats discrim-

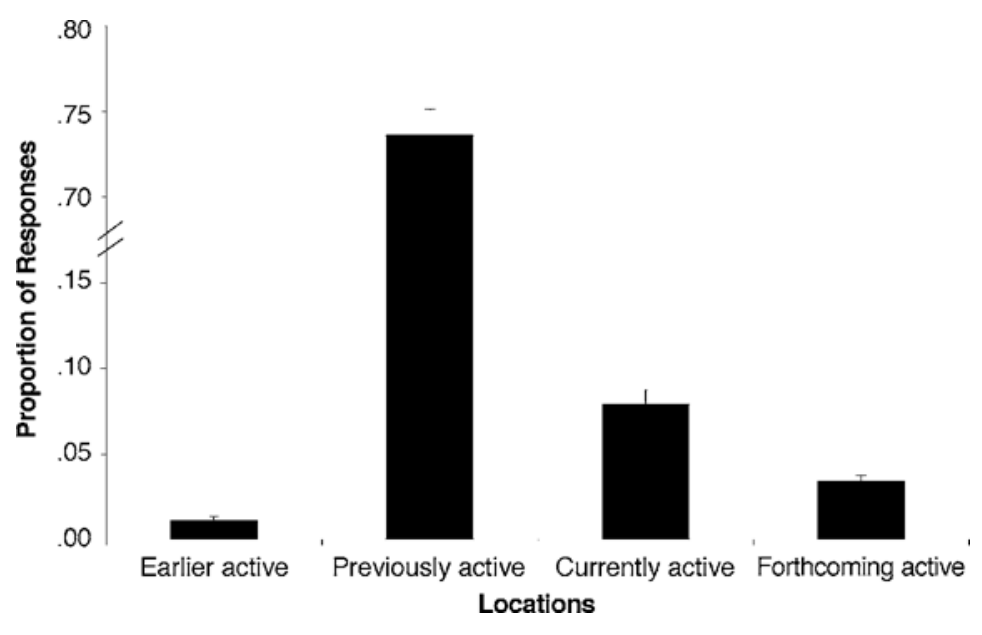

Figure 7. The average proportion of responses at each type of location averaged across Time Zone Transitions $2-8$ before food transition cues were received in Experiment 1; error bars represent $S E$. Note the break in the $y$-axis. 
inated the currently active location from the forthcoming and earlier active locations. In this respect, obtaining a nonfood cue masked knowledge of the currently active location. To assess what knowledge the rats had about which location was currently active in the absence of food transition cues, we compared the average proportion of responses at earlier active locations with that at the currently active location. The analysis revealed a significant difference $[t(3)=6.64, p<.05]$, suggesting that the rats had knowledge of which locations have provided reinforcement, as well as which location was currently active during the session. The rats allocated more responses to the currently active location than they did to the earlier active locations. This pattern of behavior suggests the use of working memory because the rats' needed to continuously update their memory for those locations that provided reward in the session, then use that information in order to search not-yet-rewarded locations (i.e., the status of earlier active locations and currently active locations changed throughout the session). The rats allocated more responses to the currently active location than they did to forthcoming active locations $[t(3)=8.3, p<.05]$. This observation suggests that the rats had information about which location was currently active.

In conclusion, although the rats had a strong tendency to remain at the previously active location at the start of a new time zone, there is evidence that the rats discriminated the currently active location from earlier active locations and from forthcoming active locations. The rats adopted a win-stay, lose-shift strategy but had additional knowledge of time and place.

Spatial choice after a nonfood cue. The previous section analyzed where the rats searched after a time zone transition occurred. It showed that the rats primarily visited the previously active location. However, it provided evidence that the rats had information about which location was currently active prior to receiving a nonfood or a food cue. To provide a converging line of evidence that the rats had information concerning the currently active location, we analyzed where the rats visited immediately after leaving the previously active location. If the rats used a winstay, lose-shift strategy with no knowledge of time and place, visits should have occurred randomly once they left the previously active location. Alternatively, if the rats had knowledge of the currently active location prior to obtaining food, the visits to the currently active location should have occurred at a rate higher than would be expected by chance.

In order to assess the rats' knowledge of the currently active location, we analyzed the first visit immediately after they left the previously active location; in this analysis, the rats may have made multiple visits to the previously active location before leaving it. If the rats used working memory to keep track of previously rewarded locations, the number of locations to randomly choose from decreased as the session progressed. Consequently, chance performance increased as a function of time zones as the number of locations excluded by working memory increased throughout the session (i.e., working memory er- rors were excluded from this analysis). For example, after a rat had visited the previously active location at the start of the second time zone, the number of remaining active locations during the session was seven. Thus, chance performance was 1/7. In contrast, after a rat had visited the active location at the start of the third time zone, the number of remaining active locations was six. Therefore, chance performance was $1 / 6$. Near the end of the session, chance performance at the start of Time Zone 7 was .5. The average rate of visiting a correct location averaged across Time Zones $2-7$ by chance was .27 according to this model.

The mean proportion of visits to the currently active location for Time Zones 2-7 was .41 (SE = .05). The observed proportion of visits to the currently active location was significantly above chance $[t(3)=3.14, p<.026$, one-tailed]; the test was one-tailed because the hypothesis that the rats had knowledge of time and place predicts that the rats would visit the currently active location at a rate greater than that expected by chance. A win-stay, loseshift strategy plus working memory for the previously rewarded locations, but without other knowledge of time or place, is insufficient to explain the performance of the rats. The rats apparently had knowledge of the next active location prior to obtaining food at that location.

These results suggest that a win-stay, lose-shift strategy with no knowledge of time and place is not adequate to explain the performance of the rats. If they had been using this strategy, the rats would have searched randomly among the remaining locations. The rats went to the correct location following a nonfood cue more frequently than would be expected by chance, suggesting that they were not visiting locations randomly. They were utilizing information about time and place to get to the next active location more efficiently than would be expected by chance once a time zone has ended.

\section{EXPERIMENT 2}

In Experiment 1, the transitions to the next active location were dependent on receiving a nonfood cue at the previously active location, but we concluded that the rats had knowledge about which location was currently active. In order to provide a converging line of evidence for this conclusion, we experimentally manipulated the food transition cue. If the rats know nothing about which location is active, then when food cues are removed, visits would be predicted to occur randomly among all locations. In contrast, if the rats have knowledge about the next active location, then when food cues are removed, visits to the active location will occur at a higher rate than to forthcoming locations and previously active locations.

\footnotetext{
Method

Subjects. The rats from Experiment 1 were used in Experiment 2.

Apparatus. The apparatus was the same as that in Experiment 1.

Procedure. Probe sessions were identical to training in Experiment 1 , except that reinforcement was not available during the fifth time zone. During these probes, food was available at each location except during the fifth time zone (i.e., the time zone at which rein-
} 


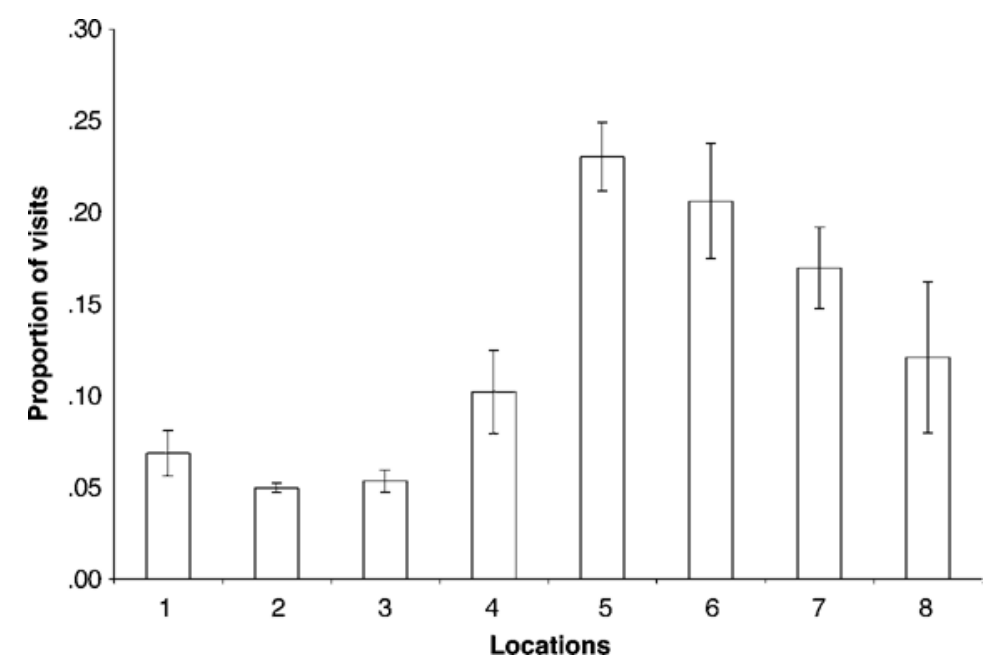

Figure 8. The proportion of visits to each location during the probe tests in Experiment 2; error bars represent $\pm 1 S E$. The data are pooled across the 7min interval in the fifth time zone.

forcement usually occurred $28-35$ min since the start of the session). Visits were counted as described in Experiment 1. Three probe sessions were conducted. Intermixed with these probe days were training days that were identical to acquisition described in Experiment 1 . Each probe was randomized within a 4-day block, with the restriction that probes were separated by at least 2 consecutive training days.

\section{Results and Discussion}

Figure 8 plots the proportion of visits to each of the locations during the probe tests. The dependent variable is the proportion of visits allocated to each location during the 7-min interval that was not rewarded in probe tests (i.e., Time Zone 5). For consistency with the terminology outlined in Experiment 1, we refer to Location 5 as currently active. However, in the probes, no reward was available. The visits do not appear to have been evenly distributed across locations. There were larger proportions at the currently active and the forthcoming active locations (i.e., Locations 5-8), relative to the earlier and the previously active locations (Locations 1-4). The location with the largest proportion of visits was the fifth location.

These data were subjected to an ANOVA with location as a repeated measure. There was a significant effect of location $[F(7,21)=8.07, p<.01]$. There was a significant difference between the proportion of responses allocated to the fifth location relative to the previously active location [i.e., Location $4 ; t(3)=3.23, p<.05$ ]. The fifth location had significantly more responses than the average of the forthcoming active locations [i.e., Locations $6-8 ; t(3)=$ $3.55, p<.05]$. There was also a significant difference between the fifth location and the average of the earlier active locations [i.e., Locations $1-3 ; t(3)=8.97, p<.01$ ]

The data suggest that the rats discriminated the fifth location from (1) the previously active location (i.e., Location 4), (2) earlier active locations (Locations 1, 2, and 3), and (3) forthcoming active locations (Locations 6, 7, and
8). The low proportion of responses at the previously active location (i.e., Location 4) likely occurred because the rats received a nonfood cue at this location (cf. Figure 7). In Experiment 2, a nonfood manipulation was used to provide a converging line of evidence for the conclusions that were reached in the analysis of the data from Experiment 1.

\section{EXPERIMENT 3}

The results of Experiment 1 showed that the rats were able to discriminate locations and times. Further analyses suggested that the rats had knowledge of the time and place at which food was available, in addition to the use of food transition cues. This conclusion was supported by the results of Experiment 2. The question remains, How were the rats solving the time-place task?

Although the rats restricted most of their visits to the active time zones, the cue(s) that they used remain to be identified. There are several cues that the rats could have used to complete the task. Presumably, multiple cues are simultaneously available during the standard training conditions. Our approach was to identify four cues that were confounded in the standard training conditions (i.e., the rats may have used one or more of these cues). To identify which, if any, of these cues were used by the rats, we separately unconfounded one cue at a time in a series of four manipulations.

The animals may have solved the time-place discrimination by using any of four mechanisms. First, active locations could have been predicted by the amount of time that had elapsed since light onset in the colony. According to this proposal, the rats reset an interval clock when the lights were turned on in the colony. Because the time of testing was carefully controlled, the interval between light onset and food availability was a reliable variable that could have been used to arrive at active locations. Second, 
the rats were handled immediately prior to testing, and the doors opened at the start of daily testing. Therefore, handling and/or the opening of the doors could have served as a cue that led the animals to the first active location. The event of being handled may be a salient cue to an animal, and a rat may use this cue as part of a stimulus-response habit (i.e., when the handling cue[s] are experienced, doing a particular response-entering Arm 1-is rewarded by food). Third, active locations could have been predicted by the amount of time that elapsed since being handled at the start of the session. According to this proposal, the rats reset an interval clock when they were handled at the start of the session. Food at each active location was available after a predictable amount of time with respect to handling. And fourth, active locations could have been predicted on the basis of time of day. Although it is unlikely that a circadian oscillator can be used to time short intervals, time of day was a reliable variable.

These four mechanisms can be summarized as follows. There are two timing mechanisms (i.e., interval and circadian). Within the interval timing mechanism, there are two potential resetting cues (i.e., light onset in the colony and handling of the rats). There is one nontiming hypothesis; the event of being handled represents a salient cue that the animal may use as part of a stimulus-response habit. In particular, when the rat is handled, doing a particular response (i.e., enter Arm 1) is followed by a reward. The distinction between the handling interval and the handling cue hypotheses is subtle. One proposal is that the rats may reset an interval clock when they are handled and time an interval with respect to this event. The alternative proposal is that the stimulus of being handled prior to testing is a cue to enter Arm 1.

The four mechanisms described above were confounded during standard training conditions. The purpose of Experiment 3 was to dissociate each of these mechanisms, one at a time. This required four manipulations. In each procedure, we adjusted one or more of the following: the time at which the colony lights were turned on, the time at which the animals were placed in the maze, and the time at which the doors to the arms of the maze were opened. These events either occurred at their training times or were adjusted by $42 \mathrm{~min}$. For each procedure, the mechanisms described above predicted that the rats would visit Location 1 or 7.

\section{Experiment 3A}

The light onset interval hypothesis was tested by turning the lights on earlier than usual in the colony and testing the rats at the standard training time. The rats would go to the location that corresponded to Time Zone 7 if they were timing with respect to light onset in the colony. The rationale for this prediction is that if they were timing the interval since light onset and we advanced light onset by $42 \mathrm{~min}$, the rats should respond at the location that is $42 \mathrm{~min}$ into the session (i.e., the location associated with Time Zone 7). Changing the time at which light onset occurs should have no affect according to the other mecha- nisms. The time of day cue has not changed because the time of day at which the session started during the test is the same as it was during training. Therefore, time of day predicts the location associated with Time Zone 1. If handling the rats and/or opening the doors at the start of a session served as a cue at the start of a session, the rats would visit Location 1. Altering the light cycle did not change the interval between handling and testing. Consequently, if the rats were timing the interval since being handled they would be predicted to go to Location 1 .

\section{Method}

Subjects. The rats from Experiments 1 and 2 were used in Experiment $3 \mathrm{~A}$.

Apparatus. The apparatus was the same as that in Experiments 1 and 2 .

Procedure. The rats received 8 days of training before this manipulation. The training was identical to that described in Experiment 1 . On the test day, light onset occurred 42 min early, relative to training conditions. Testing began at the same time as it did during training. The doors were opened immediately after placing the rat in the central hub. The duration of testing was $14 \mathrm{~min}$. Food was not available during the probe test.

\section{Data Analysis}

Because there was no reinforcement given during these procedures, visits became less numerous, and the rats began to search all locations as time into the interval progressed. Therefore, the analysis was restricted to the first $2 \mathrm{~min}$.

\section{Results and Discussion}

Figure 9A plots the proportion of visits to each of the locations during probe testing. A larger proportion of visits were observed at the location associated with Time Zone 1, relative to the other locations, including Location 7; the light onset interval hypothesis predicts Location 7, whereas Location 1 is predicted by handling/door-opening cues, handling onset interval, and time-of-day hypotheses. The data were subjected to an ANOVA with location as a repeated measure. There was a significant effect of location $[F(7,21)=10.1, p<.01]$. Locations 1 and 7 were also significantly different $[F(1,21)=58.6, p<.01]$. These data rule out a mechanism that times an interval with respect to light onset. They are consistent with the following mechanisms: timing an interval with respect to a handling event, handling/door-opening cues, and time of day.

\section{Experiment 3B}

We tested the handling/door-opening cues hypothesis by isolating the rats in the central chamber and blocking access to the arms by delaying the opening of the doors by $42 \mathrm{~min}$. If the rats were using handling/door cues, they would be predicted to go to the location associated with Time Zone 1 . They would visit this location because this is where they go whenever they are first handled or when the doors first open. The time-of-day hypothesis predicts that the rats will visit the location associated with Time Zone 7 because the current time of day corresponds to the time at which Location 7 is usually active. If the rats were timing the interval with respect to being handled, they 


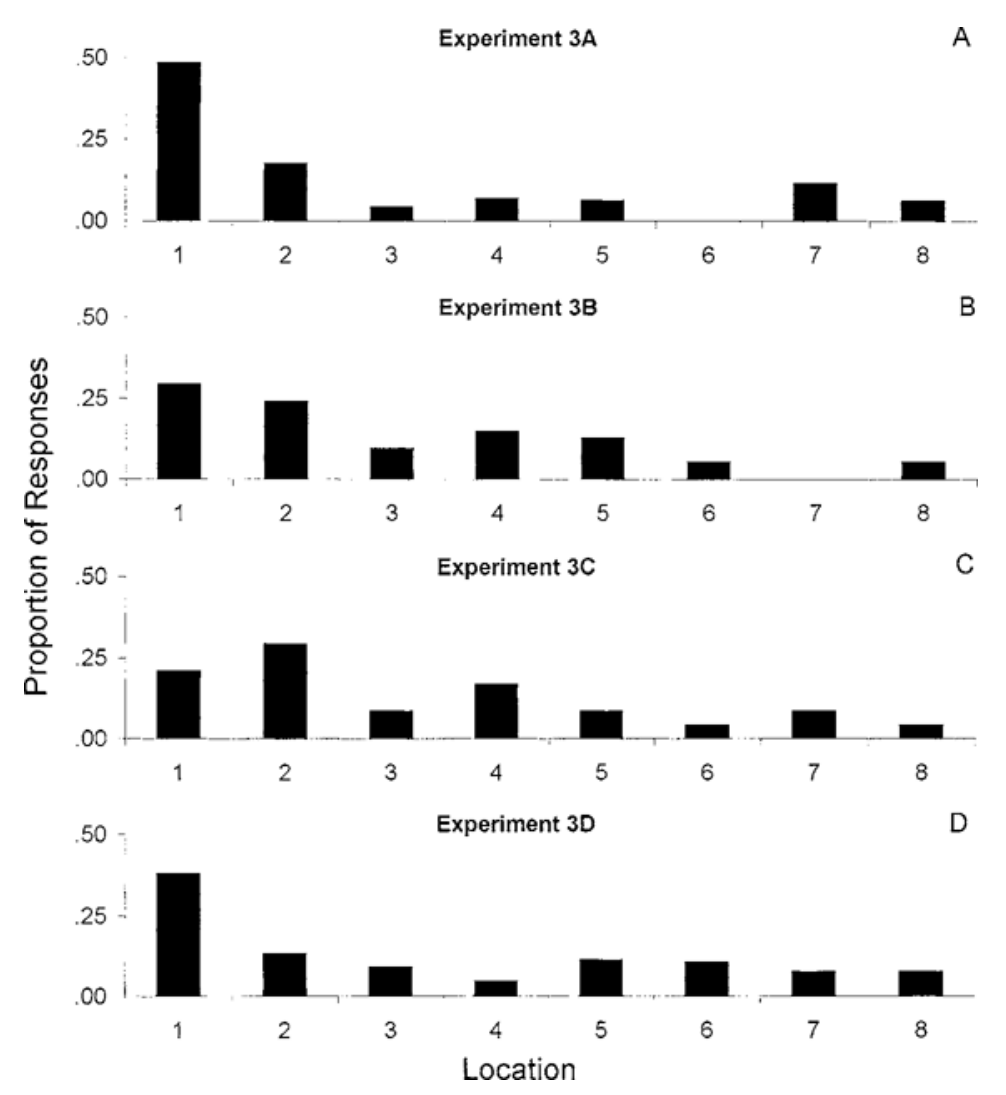

Figure 9. The proportion of visits to each location in Experiments 3A-3D. The data are pooled across the first 2 min of testing.

would be predicted to visit Location 7 , because the rats were handled and then spent the equivalent of six time zones isolated in the central chamber. If the rats were timing an interval with respect to light in the colony, they would be predicted to visit Location 7. Although the rats were isolated in the chamber for $42 \mathrm{~min}$, the interval of time between light onset and $42 \mathrm{~min}$ into the session corresponded to Time Zone 7.

\section{Method}

Subjects, Apparatus, and Data Analysis. The subjects, apparatus, and data analysis were the same as those described in Experiment 3A.

Procedure. The rats received 16 days of training before this manipulation. On the test day, the rats were placed in the central chamber of the radial maze at the same time as they were during training. Access to the arms was blocked for $42 \mathrm{~min}$. In all other respects, the procedure was the same as that in Experiment 3A.

\section{Results and Discussion}

Figure 9B plots the proportion of visits to each of the locations. The proportion of visits during the probe was largest at the location associated with Time Zone 1, relative to the other locations, including Location 7. The handling/dooropening cue hypothesis predicts Location 1, whereas Location 7 is predicted by light onset interval, handling onset interval, and time-of-day hypotheses. An ANOVA with repeated measures revealed a significant effect of location $[F(7,21)=5.5, p<.01]$. Locations 1 and 7 were also significantly different $[F(1,21)=47.8, p<.01]$.

These data rule out a mechanism that times an interval with respect to light onset or handling onset and one that uses time of day. The data are consistent with a mechanism that uses handling/door-opening cues. This suggests that the rats initiated their search on the basis of a conditional discrimination; immediately after being handled, the rats visited Location 1; the rats arrived at other locations by timing successive 7 -min intervals.

\section{Experiment 3C}

In order to test whether the rats were timing an interval with respect to the time at which handling occurred, testing was advanced, and the opening of the doors was delayed by blocking access to the arms for $42 \mathrm{~min}$. If the rats were timing the interval since handling, they would visit the location associated with Time Zone 7 . Because access to the arms was delayed by $42 \mathrm{~min}$, the interval between handling and the opportunity to visit a location corresponds to the amount of time that ordinarily occurs prior to Time Zone 7. Advancing the start of testing and delaying the opening of the doors should have no effect ac- 
cording to the other mechanisms. The use of handling/ door-opening cues predicts Location 1, because this is the first location to visit when the arms are first accessible. Although the rats were placed in the testing apparatus earlier and isolated in the central chamber for $42 \mathrm{~min}$, the interval between light onset and testing corresponds to the value that ordinarily occurs during Time Zone 1 . The opportunity to respond during this manipulation occurs at the time of day that corresponds to the first time zone, because the advance in handling and the delay in opening the doors cancel each other out.

\section{Method}

Subjects, Apparatus, and Data Analysis. The subjects, apparatus, and data analysis were identical to those described in Experiment 3A.

\section{Procedure}

The rats received 8 days of training before this manipulation. On the test day, the start time for each animal was advanced by $42 \mathrm{~min}$ (i.e., the rats were handled earlier than in training). After a rat was placed in the central chamber, the doors remained closed for $42 \mathrm{~min}$. In all other respects, the procedure was the same as that in Experiment $3 \mathrm{~A}$.

\section{Results and Discussion}

The proportion of visits to all locations are plotted in Figure $9 \mathrm{C}$. The proportions of visits are larger at the locations associated with Time Zones 1 and 2, relative to the other locations. The handling onset interval hypothesis predicts Location 7, whereas Location 1 is predicted by light onset interval, time-of-day, and handling/door cue hypotheses. These data were subjected to an ANOVA with location as a repeated measure. This revealed a significant effect for location $[F(7,21)=3.0, p<.05]$. Locations 1 and 7 were also significantly different $[F(1,21)=5.9, p<.05]$.

These data rule out a mechanism that times an interval with respect to handling. These data are consistent with the following mechanisms: time of day, handling/dooropening cues, and timing an interval with respect to light onset in the colony.

\section{Experiment 3D}

Testing the time-of-day hypothesis involved delaying the time at which light onset took place in the colony room and delaying the handling of the rats by $42 \mathrm{~min}$. If the rats were visiting locations on the basis of time of day, the rats would be predicted to visit Location 7. Delaying light onset and delaying handling of the rats should have no effect according to the other mechanisms. Because access to the arms was delayed by $42 \mathrm{~min}$, the doors opened at the time of day that corresponded to Time Zone 7. The use of handling/door-opening cues would predict Location 1, because this would be the first location to visit when the arms were first accessible, regardless of the time at which handling occurred or the light onset change. Because the doors opened immediately after the rats were handled, the interval between handling and responding corresponded to the interval associated with Location 1 . Therefore, rats timing an interval with respect to handling would be predicted to visit Location 1. Because the delay of colony lights and the delay in handling the rats canceled out one another, the interval between colony light onset and the opportunity to visit locations was identical to the amount of time that ordinarily occurred prior to Time Zone 1 . Therefore, if the rats were timing with respect to light onset, they would be predicted to visit Location 1 .

\section{Method}

Subjects, Apparatus, and Data Analysis. The subjects, apparatus, and data analysis were identical to those described in Experiment 3A.

Procedure. The rats received 9 days of training before this manipulation. On the test day, light onset occurred 42 min later, relative to training days. The start time for each animal was 42 min later than during the training days. The doors opened immediately after the rat was placed in the central hub. In all other respects, the procedure was the same as that in Experiment 3A.

\section{Results and Discussion}

Figure 9D plots the proportion of visits at all locations during probe testing. The proportion is largest at the location associated with Time Zone 1. The time-of-day hypothesis predicts Location 7, whereas Location 1 is predicted by the handling onset interval, light onset interval, and handling/door-opening hypotheses. These data were subjected to an ANOVA with location as a repeated measure. There was a significant effect of location $[F(7,21)=6.9$, $p<.01]$. There was also a significant difference between Locations 1 and $7[F(1,21)=57.8, p<.01]$.

These data rule out a mechanism that uses time of day as a cue. The data are consistent with the following mechanisms: timing an interval with respect to handling or light onset and using handling/doors-opening as cues.

In conclusion, the results of Experiment 3 are consistent with the handling/door-opening cue hypothesis. In particular, the rats used the handling and/or the opening of the doors as a cue to visit the first location. The results were consistent with this hypothesis when this was dissociated from the other hypotheses (Experiment 3B) and when the handling/door cue hypothesis was not dissociated from the other hypotheses (Experiments 3A, 3C, and 3D). In contrast, no other hypothesis is able to account for all the results of Experiments 3A-3D. These results suggest that the rats visited Location 1 by discriminating handling/ doorsopening cues at the start of the session. Next, they timed successive 7-min intervals to visit each subsequent location.

\section{GENERAL DISCUSSION}

Rats were tested in a time-place task in an eight-arm radial maze. The location of food availability changed every $7 \mathrm{~min}$. The rats restricted the majority of their visits to the locations that were active during the session (Figures 1 and 2). However, this pattern could be produced without the rats having any knowledge of time or place. The rats may have searched the maze until they found the location that was currently providing food, stayed at that location 
until food was no longer available, and then searched again to find the next location with food. However, if the rats had no knowledge of time and place, they should have searched randomly after leaving the previously active location. There are six lines of evidence to suggest that the rats had knowledge of time and place:

First, the rats anticipated the arrival of the next active location (Figure 3 ) before food became available at that location; the response rate at the next correct location increased as the next time zone approached, before food became available at that location. In contrast, the response rate at other inactive locations was relatively low and flat. These observations suggest that the rats were timing the arrival of food at the next correct location. A rat with no knowledge of time and place would not anticipate the arrival of the next correct location.

Second, the interval being timed at the next active location was identified. The superposition (i.e., analysis of the width of the anticipation function at each location) in time relative to $7 \mathrm{~min}$, rather than in elapsing time $(7,14$, $21, \ldots 49 \mathrm{~min})$, suggests that the rats were timing successive 7-min intervals (Figure 4). A rat with no knowledge of time and place would not time the interval.

Third, the rats anticipated the end of the currently active location (Figure 5); the response rate at the currently active location decreased as the end of the time zone approached, before food at the currently active location had ended. This observation is consistent with the hypothesis that the rats were timing successive 7 -min intervals. A rat with no knowledge of time and place would not anticipate the end of the current time zone.

Fourth, at the start of a new time zone (i.e., before the rat had obtained a nonfood cue at the previously active location and before the animal had obtained a food cue at the currently active location), the animals visited the currently active location at a rate that was higher than that for visits to the forthcoming active and earlier active locations (Figure 7). A rat with no knowledge of space and time would be expected to visit these three types of locations at the same rates.

Fifth, on nonrewarded probe tests (Experiment 2), the rats visited the currently active location (in the absence of food cues) more than they did forthcoming and earlier locations (Figure 8). A rat with no knowledge of space and time would be expected to visit these three types of locations at the same rates.

And sixth, after the rats had left the previously active location, they visited the next correct location at a rate higher than would be expected by chance. The model of chance assumed random selection of all arms except any arm that was previously rewarded earlier in the session (i.e., working memory). A rat with no knowledge of space and time would be expected to visit the next correct location at the chance level. In contrast, the rats anticipated the next active location, which suggests that they had knowledge of the next active location prior to receiving a food cue.

Experiment 3 was conducted in order to identify the mechanism that enabled the rats to complete the time- place task. We identified four mechanisms that may have been used to restrict visits to the temporally and spatially correct locations: (1) The rats may have been timing the interval since light onset in the colony, (2) the rats may have timed the interval since being handled at the start of the session, (3) the rats may have been using time of day, and (4) the rats may have used handling/doors-opening as a cue to go to the first location. These four mechanisms were confounded during training conditions. In Experiment 3 , we systematically unconfounded each mechanism. The handling/doors-opening hypothesis was consistent with the results of all four tests in Experiments 3A-3D. It was concluded that the rats were using the handling event or the opening of the doors at the start of the session as a cue to go to the first location.

We propose the following model of the rats' behavior in the time-place task. The rats used the handling event and/ or the opening of the doors as a cue to go to the first location. Next, the rats timed successive 7-min intervals at each active location. The rats were also sensitive to food transition cues. In summary, the rats had a detailed representation of time and place: The rats had knowledge of where to start, where they had already received reward, and where they need to go next, and they timed the interval of reinforcement at each location.

The mechanism that is responsible for identifying the next active location remains to be identified. One possibility is that the rats have knowledge of the sequence of active locations. Another possibility is that the rats use a stimulus-response chain that enables them to get to the next location from each previous location. There is no evidence to support the proposal that the animals developed an association between time of day and location.

This study differs from other time-place studies in several ways. First, it increased the spatial demands of a timeplace task by including eight locations. Second, the time of testing was carefully controlled, thereby making interval and time-of-day cues available. Third, the magnitude of anticipation was relatively small, as compared with other time-place studies; small anticipatory changes reflected a low error rate, suggesting that the rats checked the next active location while minimizing the lost opportunity to obtain food at the currently active location. And fourth, we compared performance with a model of random behavior to establish knowledge of time and place.

In conclusion, the results from the present study suggest that the rats met the time-place contingency by using an interval-timing mechanism. Although time of day was a reliable cue, the rats did not use it. There are two potential explanations for this outcome. First, 7 min may have been too short an interval to support circadian time-place discrimination. Intervals in the minutes range are compatible with the use of an interval timer. Second, discriminating between eight phases of a circadian oscillator may exceed the limits of the circadian system. This limit may be based on the number of phases that may be discriminated with a circadian system or on the number of circadian oscillators that may be entrained to each rewarded location. 


\section{REFERENCES}

Aschoff, J. (1984). Circadian timing. In J. Gibbon \& L. Allan (Eds.), Timing and time perception (Annals of the New York Academy of Sciences, Vol. 423, pp. 442-468). New York: New York Academy of Sciences. AsCHOFF, J. (1985). On the perception of time during prolonged temporal isolation. Human Neurobiology, 4, 41-52.

Aschoff, J. (1989). Temporal orientation: Circadian clocks in animals and humans. Animal Behaviour, 37, 881-896.

Aschoff, J. (1993). On the passage of subjective time in temporal isolation. Psychologica Belgica, 33, 147-157.

AsCHOFF, J. (1998). Human perception of short and long time intervals: Its correlation with body temperature and the duration of wake time. Journal of Biological Rhythms, 13, 437-442.

AsCHOFF, J., \& DAAN, S. (1997). Human time perception in temporal isolation: Effects of illumination intensity. Chronobiology International, 14, 585-596.

BiEbach, H., FALK, H., \& Krebs, J. R. (1991). The effect of constant light and phase shifts on a learned time-place association in garden warblers (Sylvia borin): Hourglass or circadian clock? Journal of Biological Rhythms, 6, 353-365.

Biebach, H., GoRdiJN, M., \& KREBS, J. R. (1989). Time and place learning by garden warblers, Sylvia borin. Animal Behaviour, 37, 353-360.

CARR, J. A. R., \& Wilkie, D. M. (1997). Rats use an ordinal timer in a daily time-place learning task. Journal of Experimental Psychology. Animal Behavior Processes, 23, 232-247.

CARR, J. A. R., \& WILKIE, D. M. (1998). Characterization of the strategy used by rats in an interval time-place learning task. Journal of Experimental Psychology: Animal Behavior Processes, 24, 151-162.

CRYstal, J. D. (2001). Circadian time perception. Journal of Experimental Psychology: Animal Behavior Processes, 27, 68-78.

CRYSTAL, J. D. (2003). Nonlinearities in sensitivity to time: Implications for oscillator-based representations of interval and circadian clocks. In
W. H. Meck (Ed.), Functional and neural mechanisms of interval timing (pp. 61-75). New York: CRC Press.

CRYSTAL, J. D. (2004). Sensitivity to time: Implications for the representation of time. Manuscript submitted for publication.

Crystal, J. D., \& Miller, B. J. (2002). Simultaneous temporal and spatial processing. Animal Learning \& Behavior, 30, 53-65.

DaAn, S., \& Koene, P. (1981). On the timing of foraging flights by oystercatchers, Haematopus ostralegus, on tidal mudflats. Netherlands Journal of Sea Research, 15, 1-22.

FrISCH, K. VON (1967). The dance language and orientation of bees. (E. Lei gh, Trans.). Cambridge, MA: Harvard University Press, Belknap Press.

Gibbon, J. (1991). Origins of scalar timing. Learning \& Motivation, 22, 3-38.

GibBON, J., FAIRHURST, S., \& GoldBERG, B. (1997). Cooperation, conflict and compromise between circadian and interval clocks. In C. M. Bradshaw \& E. Szabadi (Eds.), Time and behavior: Psychological and neurobehavioral analyses (pp. 329-384). New York: Elsevier.

Krebs, J. R., \& BIEBACH, H. (1989). Time-place learning by garden warblers (Sylvia borin): Route or map? Ethology, 83, 248-256.

Mistlberger, R. E., De Groot, M. H. M., Bossert, J. M., \& MarCHANT, E. G. (1996). Discrimination of circadian phase in intact and suprachiasmatic nuclei-ablated rats. Brain Research, 739, 12-18.

Pizzo, M. J., \& Crystal, J. D. (2002). The representation of time in time-place learning. Animal Learning \& Behavior, 30, 387-393.

SAKSIDA, L. M., \& WILKIE, D. M. (1994). Time-of-day discrimination by pigeons, Columba livia. Animal Learning \& Behavior, 22, 143-154.

WAHL, O. (1932). Neue Untersuchungen über das Zeitgedächtnis der Bienen. Zeitschrift für vergleichende Physiologie, 16, 529-589.

(Manuscript received October 28, 2003; revision accepted for publication January 6,2004 .) 